And then I felt sorry. I don't want to hurt anybody and especially I don't want to hurt poor Emmy Noether. I thought about it repeatedly and finally I decided that, after all, it was not one hundred per cent my fault. She should have answered: "And a mathematician who can only specialise is like a monkey who can only climb DOWN a tree."

In fact, neither the up, nor the down, monkey is a viable creature. A real monkey must find food and escape his enemies and so he must incessantly climb up and down, up and down. A real mathematician must be able to generalise and to specialise.

A particular mathematical fact behind which there is no perspective of generalisation is uninteresting. On the other hand, the world is anxious to admire that apex and culmination of modern mathematics: a theorem so perfectly general that no particular application of it is possible.

There is, I think, a moral for the teacher. A teacher of traditional mathematics is in danger of becoming a down monkey, and a teacher of modern maths an up monkey. The down teacher dishing out one routine problem after the other may never get off the ground, never attain any general idea. And the up teacher dishing out one definition after the other may never climb down from his verbiage, may never get down to solid ground, to something of tangible interest for his pupils. It seems to me that the quality of disservice to the pupils and to the taxpayers is very much the same in both cases.

What is desirable? To look for generality behind the particular case, to look for significant particular cases in the general statement. "The union of passionate interest in the detailed facts with equal devotion to abstract generalisation" said A. N. Whitehead ([1], p. 3).

\title{
Reference
}

1. A. N. Whitehead, Science and the Modern World. Cambridge University Press (1926).

Stanford University, California 94306, USA

G. POLYA

\section{Triangulated polygons and frieze patterns}

\section{J. H. CONWAY AND H. S. M. COXETER}

\section{Introduction}

By a triangulated $n$-gon we mean a partition of a polygon $P_{0} P_{1} \ldots P_{n-1}$ (not necessarily regular) into $n-2$ triangles by means of $n-3$ non-crossing diagonals. Let $a_{r}$ denote the number of triangles at $P_{r}$. Clearly

$$
a_{0}+a_{1}+\cdots+a_{n-1}=3(n-2), \quad 1 \leqslant a_{r} \leqslant n-2,
$$

and if $n>3$, at least two of the as are equal to 1 but no two consecutive as can take this value. While developing a set of problems and solutions, we 
shall find other properties of this cycle of numbers and exhibit it as the second row of a frieze pattern

$$
\begin{array}{lllllllllll}
1 & & 1 & & 1 & \ldots & 1 & & 1 & & 1 \\
& a_{0} & & a_{1} & & \ldots & & a_{n-1} & & a_{0} & \\
& & \ldots & & & \ldots & & & \ldots & &
\end{array}
$$

But let us first decide what we shall mean by a "frieze pattern".

\section{Problems about frieze patterns}

From the standpoint of symmetry, every infinitely long frieze belongs to one of seven types ([12], p. 88):

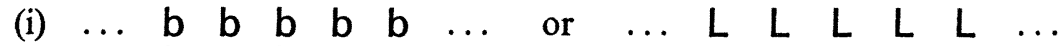

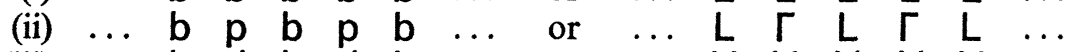

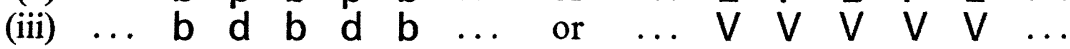

$$
\begin{aligned}
& \text { (iv) } \ldots \begin{array}{llllllllllllllllll}
\ldots & \ldots & & b & q & b & \ldots & \text { or } & \ldots & N & N & N & N & N & \ldots
\end{array}
\end{aligned}
$$

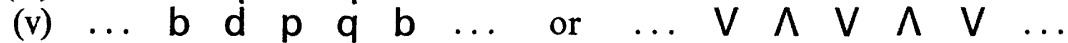

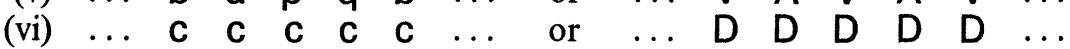

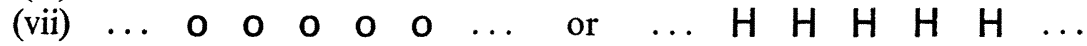

All these patterns can be shifted a certain distance to the right or left without being changed: they are symmetrical by translations (or slides). In case (i) these are the only symmetries. But in case (iii) there are also reflections (or flips) about some vertical lines, and in case (iv) there are some half-turns: the pattern looks the same when the page has been turned upside down. In case (v) there are both reflections and half-turns. The combination or product of a reflection and a half-turn is a glide (which takes the $b$ to the $p$ ) like the relation between successive footprints when one walks along a straight path covered with snow. This glide appeared by itself (without the component reflection and half-turn) in case (ii). Case (vi) is like (i) but with an extra reflection (flip) about a horizontal line.

(1) What happens in case (vii)?

(2) Which are the types of the following patterns of Roman numerals?

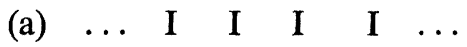

$$
\begin{aligned}
& \begin{array}{lllllllll}
\text { (c) } & & \text { I } & & \text { I } & & \text { I } & & \text { I } \\
& \ldots & \text { I } & & \text { II } & & \text { I } & & \text { II } \\
& \text { I } & & \text { I } & & \text { I } & & \text { I } & \ldots
\end{array}
\end{aligned}
$$

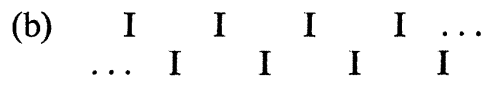

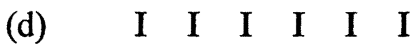

$$
\begin{aligned}
& \text {... III I II II I III } \\
& \text { II II I I III I I II ... }
\end{aligned}
$$

Roman numerals I, II, III have been used because they are symmetrical by reflections and half-turns. If we agree to overlook the 'accidental' fact that ordinary numerals lack symmetry, we can re-write (d) in the form 


$\begin{array}{rrrrrrrrrrrr}1 & & 1 & & 1 & & 1 & & 1 & & 1 & \ldots \\ \ldots & 3 & & 1 & & 2 & & 2 & & 1 & & 3 \\ 2 & & 2 & & 1 & & 3 & & 1 & & 2 & \ldots \\ \ldots & 1 & & 1 & & 1 & & 1 & & 1 & & 1\end{array}$

and regard it still as being of type (v).

Of which types are the following three patterns?

(3)

(4)

\begin{tabular}{|c|c|c|c|c|c|c|c|c|}
\hline 1 & 1 & & 1 & & 1 & & 1 & \\
\hline & & 3 & & 1 & & 3 & & 1 \\
\hline & 2 & & 2 & & 2 & & 2 & \\
\hline & & 1 & & 3 & & 1 & & 3 \\
\hline
\end{tabular}

$(5)$

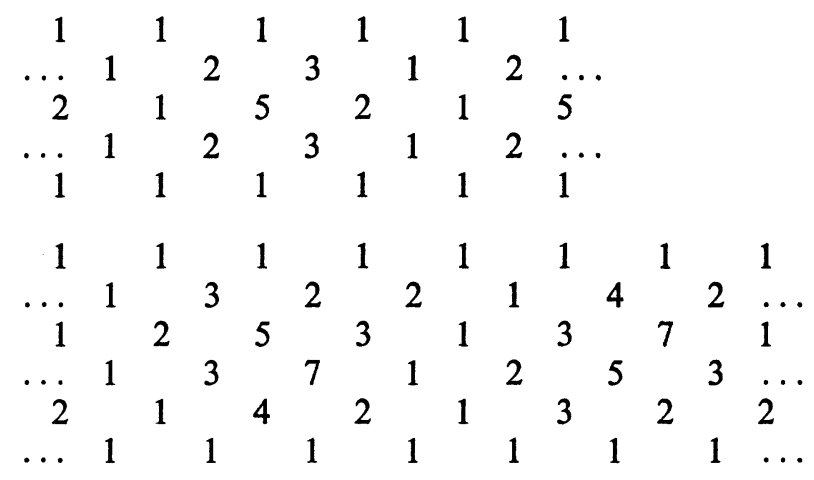

(6) What arithmetical rule (concerning multiplication and addition) is satisfied by each of these numerical patterns? Hint: Look at the 'diamond' shapes such as

$$
\begin{array}{llllll}
5 & & & & 5 \\
7 & 3 & & 7
\end{array}
$$

This rule may conveniently be called the unimodular rule.

(7) How should the pattern

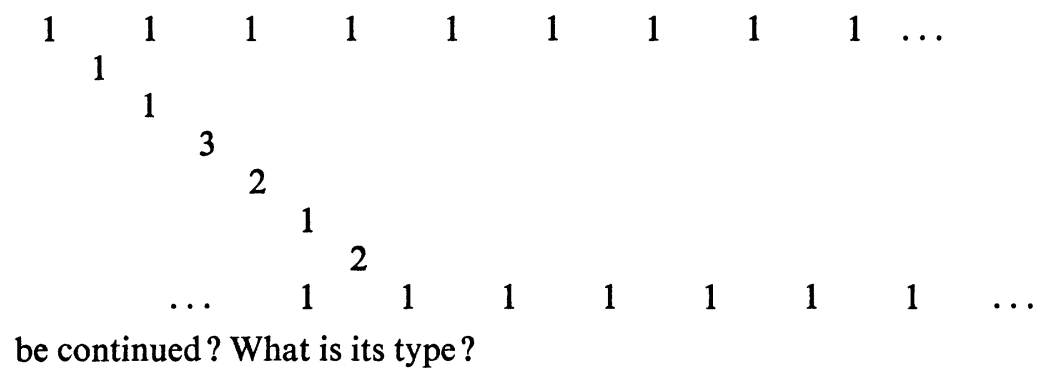


For a reason that will soon become clear, a pattern with $n-1$ rows (the first and last consisting entirely of ones) is said to be of order $n$. Thus (3) and (4) are of order six, (5) is of order seven, and (7) is of order nine. Trivially, a single row of ones, as in 2(a), may be called a pattern of order two.

(8) What does Gauss's identity

$$
\alpha\{(1+\varepsilon-\beta \gamma)-(1+\beta-\delta \varepsilon)\}=(1+\gamma)(1+\delta-\alpha \beta)-(1+\delta)(1+\gamma-\varepsilon \alpha)
$$

tell us about the pattern of order five that begins

([5], p. 298)?

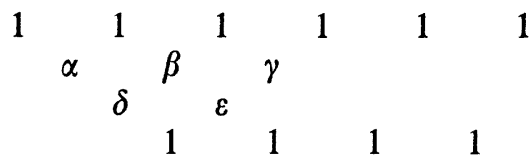

(9) The structure of the pattern

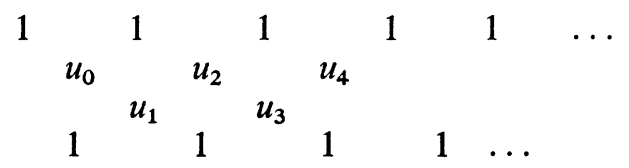

(where every $u_{r} \neq 0$ ) suggests that the equations [10]

$$
u_{0} u_{2}=1+u_{1}, \quad u_{1} u_{3}=1+u_{2}, \quad u_{2} u_{4}=1+u_{3}, \quad u_{3} u_{5}=1+u_{4}
$$

imply $u_{0}=u_{5}$. Give a simpler proof than Gauss's (all on one line).

(10) Is a frieze pattern of positive numbers determined as soon as one diagonal is filled up?

(11) If the restriction to positive numbers is temporarily abandoned, how should a frieze pattern be extended by two extra rows above, and two extra rows below, so that the unimodular rule continues to hold?

(12) If the top row of ones is still called the first row, can it happen that two consecutive ones occur also in the second row?

(13) In a frieze pattern

$\begin{array}{llllllllll}1 & & 1 & & 1 & & 1 & & 1 & \\ & a_{0} & & a_{1} & & a_{0} & & a_{1} & & \ldots\end{array}$

of order four, can it happen that $a_{0}=a_{1}$ ? 
(14) If

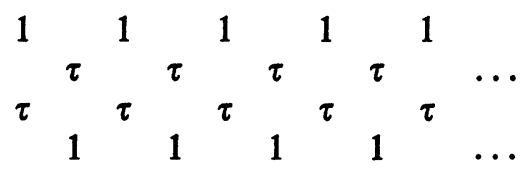

is a frieze pattern (of order five), what is the value of $\tau$ ? Have you seen this number in any other connection?

(15) It is proved in books on geometry that, when a simple quadrangle $A B C D$ is inscribed in a circle, its sides and diagonals satisfy Ptolemy's theorem

$$
A B \times C D+B C \times D A=A C \times B D
$$

([14], p. 206). What does this tell us about the diagonal $A C(=B D)$ of a regular pentagon $A B C D E$ of side $A B=1$ ?

(16) Form the analogous frieze pattern based on the sides and diagonals of a regular hexagon.

Remark. As a limiting case, one may consider the infinitely tall pattern

$\begin{array}{cccccccccc}1 & & 1 & & 1 & & 1 & & 1 & \\ & 2 & & 2 & & 2 & & 2 & & \ldots \\ 3 & & 3 & & 3 & & 3 & & 3 & \\ & 4 & & 4 & & 4 & & 4 & & \ldots \\ 5 & & 5 & & 5 & & 5 & & 5 & \\ & & \ldots & & & & \ldots & & & \ldots\end{array}$

of type (iii).

(17) Returning to the general frieze pattern of order $n$, let the numbers in the second row be

$$
a_{0}, a_{1}, a_{2}, \ldots
$$

and let those in the 'south-east' diagonal through $a_{0}$ be

$$
f_{-1}=0, f_{0}=1, f_{1}=a_{0}, \ldots, f_{n-2}=1, f_{n-1}=0,
$$

so that the pattern begins with

$$
\begin{array}{lllllllllllll}
1 & & 1 & & 1 & & 1 & \ldots & 1 & & 1 & & 1 \\
& f_{1} & & a_{1} & & a_{2} & & \ldots & & a_{n-1} & & a_{n} & \\
& & f_{2} & & & & & & & & & & \\
& & \ldots & & & & & & & & & & \\
& & & & f_{n-3} & & & & & & & \\
& & & & 1 & & 1 & & & & 1 & & 1
\end{array}
$$

Express $a_{s}$ as a function of the $f \mathrm{~s}$.

(18) Express $f_{r}$ as a function of $a_{0}, a_{1}, \ldots, a_{n-4}$. 
(19) The unimodular rule tells us that

$$
\left|\begin{array}{cc}
a_{r-1} & 1 \\
1 & a_{r}
\end{array}\right|>0
$$

except when every $a_{r}=1$. What analogous inequalities hold for sequences of three or more $a$ s?

(20) What equation relates $a_{0}, a_{1}, \ldots, a_{n-3}$ ?

(21) In an obvious sense, the pattern (3) is of period two, while (7) is of period three and (14) is of period one. We see from (9) and (14) that every pattern of order five is of period one or five. Is it always true that $a_{n}=a_{0}$ ? In other words, does every pattern of order $n$ have period $n$ or a divisor of $n$ ?

(22) For which values of $n$ is the period always strictly less than $n$ ?

(23) Suppose ...t $u v w \ldots$ is part of the second row (cycle of $a$ ) of a frieze pattern of order $n$. What happens if the cycle is changed so that this part is replaced by $\ldots t u+11 v+1 w \ldots$ ?

(24) What relation (involving addition and divisibility) must hold for any three consecutive entries in a diagonal (such as $f_{r-1}, f_{r}, f_{r+1}$ ) if the pattern is to consist entirely of integers?

\section{Problems about triangulated polygons}

Let us now carefully consider the problem of finding all possible cycles of integers $a_{r}$ that yield frieze patterns of integers. Lacking any perfectly descriptive name, we will call them quiddity cycles.

(25) Does every quiddity cycle include at least one 1 ?

(26) Can every quiddity cycle of order $n+1$ be derived from one of order $n$ by means of the rule described in (23)?

(27) Applying rule (23) to the 'cycle' 00 of order two, we obtain 111 of order three, then 1212 of order four, 13122 of order five,

$$
141222,132132,131313,231231
$$

of order six, and so on. Have you seen such cycles in any other connection?

(28) Is there just one frieze pattern of integers for each triangulated polygon?

(29) Is there just one triangulated polygon for each frieze pattern of integers? 
(30) Draw the triangulated enneagon corresponding to the frieze pattern (7). Why is this interesting when regarded as a graph?

(31) Is the number of integral frieze patterns finite for each value of $n$ ?

(32) Let a frieze pattern of integers be bordered by two rows of $P$ s, thus:

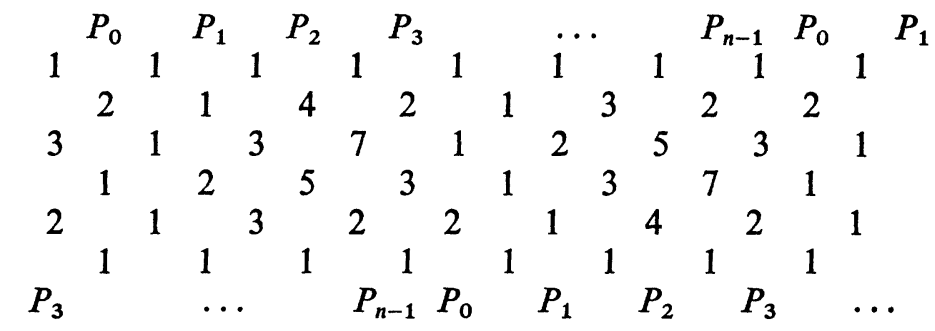

and let $(r, s)$ denote the entry where a south-east diagonal $P_{r} P_{r}$ meets a north-east diagonal $P_{s} P_{s}$, or vice versa. What happens to the polygon $P_{0} P_{1} \ldots P_{n-1}$ whenever $(r, s)=1$ ?

(33) Does the 'fundamental region'

$$
\begin{array}{lllllllll}
2 & & 1 & & 4 & & 2 & & 1 \\
& 1 & & 3 & & 7 & & 1 \\
& & 2 & & 5 & & 3 & & \\
& & & 3 & & 2 & & &
\end{array}
$$

(which is repeated upside down, between the upper and lower rows of ones) always contain just $n-3$ ones and the same number of twos?

(34) One simple way to triangulate a polygon is by means of diagonals forming a zigzag. Does this yield a frieze pattern consisting entirely of Fibonacci numbers?

(35) Can we go one step farther and assert that every frieze pattern of integers either contains a 4 or consists entirely of Fibonacci numbers?

[Section 4 (Solutions) will appear in the next issue of the Gazette. The references given below apply also to that section.]

\section{References}

1. J. Böhm, Zu Coxeters Integrationsmethode in gekrümmten Räumen, Math. Nachr. 27, 179-214 (1964).

2. W. G. Brown, Historical note on a recurrent combinatorial problem, Am. math. Mon. 72, 973-977 (1965).

3. H. S. M. Coxeter, On Schläfli's generalization of Napier's Pentagramma Mirificum, Bull. Calcutta math. Soc. 28, 123-144 (1936). 
4. H. S. M. Coxeter, Cyclic sequences and frieze patterns, Vinculum 8, 4-7 (Melbourne, 1971).

5. H. S. M. Coxeter, Frieze patterns, Acta arith. 18, 297-310 (1971).

6. Leonhard Euler, Commentationes geometricae 1, XVI-XVIII (Lausanne, 1953): review of A. de Segner, Enumeratio modorum, quibus figurae planae recitilineae per diagonales dividuntur in triangula.

7. H. G. Forder, Some problems in combinatorics, Mathl Gaz. XLV, 199-201 (No. 353, October 1961).

8. F. Harary, Graph Theory. Addison-Wesley (1969).

9. F. Harary, G. Prins and W. T. Tutte, The number of plane trees, K. Ned. Akad. Wetensch. Proc. A67, 319-329 (1964).

10. R. C. Lyness, Notes 1581; 1847; 2952, Mathl Gaz. XXVI, 62 (No. 268, February 1942); XXIX, 231 (No. 287, December 1945); XLV, 207-209 (No. 353, October 1961).

11. J. W. Moon and Leo Moser, Triangular dissections of $n$-gons, Can. math. Bull. 6, 175-178 (1963).

12. G. Polya, Mathematics and Plausible Reasoning, Vol. 1: Induction and Analogy in Mathematics. Princeton University Press (1954).

13. S. Prešić and D. S. Mikrinović, Sur une équation fonctionelle cyclique d'ordre supérieure, Publikacijc elektroteh. Fak. Univ. Beogr., Ser. Math.-Phys. 70 (1962).

14. B. L. van der Waerden, Science Awakening. Oxford University Press (New York, 1961).

Sidney Sussex College, Cambridge

University of Toronto, Toronto MSS 1A1, Canada H. S. M. COXETER

\section{Byron's daughter}

\section{B. H. NEUMANN}

$$
\begin{aligned}
& \text { But-Oh! ye lords of ladies intellectual } \\
& \text { Inform us truly, have they not hen-pecked you all? } \\
& \text { Byron: Don Juan I, xxii }
\end{aligned}
$$

These lines were inspired by Byron's wife [1]; they were married in January 1815 , and soon after they were estranged and, early in 1816, separated. Lady Byron was, in her own estimation as in her husband's, a mathematician. She learnt her mathematics from William Frend [2], who taught her astronomy, algebra, Latin, and geometry [3], and whose favourite pupil she was.

On 10 December 1815 Lady Byron gave birth to a daughter, Ada Augusta, Lord Byron's only legitimate child. Ada Byron, like her mother, studied mathematics, helped mainly by Augustus De Morgan, the first Professor of Mathematics at University College, London [4]. At the age of 19 Ada married William, Lord King, who was then 30, and who was soon after created the 1st Earl of Lovelace. Lady Lovelace had two sons and a daughter, and died, apparently of cancer and in very great pain, in 1852, at the age of 36 [5]. According to Mrs. De Morgan [6], "her mathematical 